\title{
Characterisation and Defect Analysis of 2D Layered Ternary Chalcogenides
}

Tigran Simonian ${ }^{1}$, Ahin Roy $^{1}$, Valeria Nicolosi ${ }^{2}$ and Zdeněk Sofer ${ }^{3}$

${ }^{1}$ Trinity College Dublin, United States, ${ }^{2}$ Centre for Research on Adaptive Nanostructures and Nanodevices (CRANN) and Advanced Materials and Bioengineering Research (AMBER) Research Centers, Trinity College Dublin, Dublin 2, Ireland, United States, ${ }^{3}$ University of Chemistry and Technology Prague, United States

TlGaX2 $[\mathrm{X}=\mathrm{Se}, \mathrm{S}, \mathrm{Te}]$ is a family of layered 2-D ternary chalcogenides. These p-type semiconductors have band gaps within the green to ultraviolet range [1] [2] [3], which makes them ideal candidates for optoelectronic applications.

Current examples of such applications, such as phototransistors [4] and detectors [5] [6], make use of multistep processes, such as mechanical exfoliation with a PMMA transfer [4], or thin film synthesis via thermal evaporation [6], which makes potential future scalability of these devices cumbersome. Liquid phase exfoliation (LPE) is a far more facile process that has been shown to work for a large number of layered van der Waals materials [7]. Herein, we demonstrate that these TlGaX2 materials can be exfoliated with a facile one step sonication in IPA, leaving behind little to no residue.

While there have been a number of studies into the electronic structure of these materials, an exact description of their band structures has yet to be established [6]. Interestingly, despite defect quantification being an important structure-property relationship in semiconductors, none of its aspects, e.g. stoichiometry, charge states, structural defects, etc., have yet been fully addressed. For example, while it was reported that Se vacancies in TlGaSe2 can lead to a change in the nature and size of the bandgap, a quantitative relationship was not determined 1 Structural defects that are ubiquitous in 2D materials have demonstrated to exert significant impacts on the materials' properties. In the past several decades, 2D TMDs of group VIB metal MX2, like $\mathrm{M}=\mathrm{Mo}, \mathrm{W}$, and $\mathrm{X}=\mathrm{S}$, Se, typically with hexagonal $2 \mathrm{H}$ polytype, have been studied intensively in fundamental structures, properties, and applications. They possess sizable band gaps in the range of 1.5-2.0 eV,2 corresponding to the red to near infrared regions. While the wider range of optoelectronic applications require further exploration of novel 2D materials for full cover of the colour regions. The emerging noble metal TMDs, e.g. PtX2, have recently gained a lot of attention for expanding the 2D family, due to their fascinating properties such as the layer-controllable transition from metal to semiconductor, the notable carrier mobility, strong interlayer interaction, anisotropy, and ultrahigh air stability.3-8

1. Manzeli, S.; Ovchinnikov, D.; Pasquier, D.; Yazyev, O. V.; Kis, A. Nat. Rev. Mater. 2017, 2, 17033.

2. Duan, X.; Wang, C.; Pan, A.; Yu, R.; Duan, X. Chem. Soc. Rev. 2015, 44, (24), 8859-8876.

3. Sajjad, M.; Singh, N.; Schwingenschlögl, U. Appl. Phys. Lett. 2018, 112, (4), 043101. 
4. Kandemir, A.; Akbali, B.; Kahraman, Z.; Badalov, S. V.; Ozcan, M.; Iyikanat, F.; Sahin, H. Semicond. Sci. Technol. 2018, 33, (8), 085002.

5. Zhao, Y.; Qiao, J.; Yu, Z.; Yu, P.; Xu, K.; Lau, S. P.; Zhou, W.; Liu, Z.; Wang, X.; Ji, W.; Chai, Y. Adv. Mater. 2017, 29, (5).

6. Yao, W.; Wang, E.; Huang, H.; Deng, K.; Yan, M.; Zhang, K.; Miyamoto, K.; Okuda, T.; Li, L.; Wang, Y.; Gao, H.; Liu, C.; Duan, W.; Zhou, S. Nat. Commun. 2017, 8, (1), 14216.

7. Zhao, Y.; Qiao, J.; Yu, P.; Hu, Z.; Lin, Z.; Lau, S. P.; Liu, Z.; Ji, W.; Chai, Y. Adv. Mater. 2016, 28, (12), 2399-2407.

8. Miró, P.; Ghorbani-Asl, M.; Heine, T. Angew. Chem. Int. Ed. 2014, 53, (11), 3015-3018.

9. Yu, X.; Yu, P.; Wu, D.; Singh, B.; Zeng, Q.; Lin, H.; Zhou, W.; Lin, J.; Suenaga, K.; Liu, Z.; Wang, Q. J. Nat. Commun. 2018, 9, (1), 1545.

10. Pi, L.; Li, L.; Liu, K.; Zhang, Q.; Li, H.; Zhai, T. Adv. Funct. Mater. 0, (0), 1904932.

11. Wang, Z.; Li, Q.; Besenbacher, F.; Dong, M. Adv. Mater. 2016, 28, (46), 10224-10229.

12. Wang, Z.; Wang, P.; Wang, F.; Ye, J. F.; He, T.; Wu, F.; Peng, M.; Wu, P. S.; Chen, Y. F.; Zhong, F.; Xie, R. Z.; Cui, Z. Z.; Shen, L.; Zhang, Q. H.; Gu, L.; Luo, M.; Wang, Y.; Chen, H. W.; Zhou, P.; Pan, A. L.; Zhou, X. H.; Zhang, L. L.; Hu, W. D. Adv. Funct. Mater. 2020, 30, (5), 1907945.

13. Gao, J.; Cheng, Y.; Tian, T.; Hu, X.; Zeng, K.; Zhang, G.; Zhang, Y.-W. ACS Omega 2017, 2, (12), 8640-8648.

14. Yong, X.; Zhang, J.; Ma, X. International Journal of Hydrogen Energy 2020, 45, (15), 8549-8557.

15. Avsar, A.; Cheon, C.-Y.; Pizzochero, M.; Tripathi, M.; Ciarrocchi, A.; Yazyev, O. V.; Kis, A. Nat. Commun. 2020, 11, (1), 4806. 